\title{
Doping in 2D
}

\author{
Ion implantation can be used to dope silicon devices, but can be problematic when applied to the atomically thin \\ crystal structure of two-dimensional materials - an increasing range of alternative methods is though available.
}

oping - the controlled introduction of impurities into a material in order to manipulate its properties - is an essential tool in building electronic devices. With silicon, ion implantation can be used. Here, ionized dopants are fired into a region of a semiconductor wafer, leading to an excess of electrons or holes, and thus a region of n-type or p-type material. Such techniques have played an important role in establishing silicon complementary metaloxide-semiconductor (CMOS) technology as the dominant approach in the electronics industry. Thus, in the search for alternative materials to silicon - a focus for many researchers (and many of the pages of Nature Electronics) - effective doping methods are a requirement for any contender.

For two-dimensional materials, and the layered two-dimensional materials known as van der Waals heterostructures, doping is a challenge because ion implantation can lead to damage to the atomically thin crystal structure. A range of alternative approaches are though available - and new ones continue to emerge. In last month's issue of Nature Electronics, for example, Donghun Lee, Chul-Ho Lee and colleagues reported that remote modulation doping was possible in van der Waals heterostructure transistors ${ }^{1}$. The approach uses a tungsten diselenide/hexagonal boron nitride/ molybdenum disulfide heterostructure in which the molybdenum disulfide channel can be remotely doped via controlled charge transfer from molecular dopants on the surface of the tungsten diselenide.

The researchers - who are based at Korea University, Kyung Hee University, Ulsan National Institute of Science and Technology, and the Korea Institute of Science and Technology - show that the resulting modulation-doped field-effect transistors exhibit a room-temperature mobility of $60 \mathrm{~cm}^{2} \mathrm{~V}^{-1} \mathrm{~s}^{-1}$. In comparison, molybdenum disulfide field-effect transistors that have been directly doped with the same molecular dopant (triphenylphosphine, an n-type dopant) exhibit a mobility of only $35 \mathrm{~cm}^{2} \mathrm{~V}^{-1} \mathrm{~s}^{-1}$.

Alternatively, back in our January issue, Moon-Ho Jo and colleagues reported that few-layer molybdenum ditelluride and tungsten diselenide field-effect transistors can be reversibly doped with

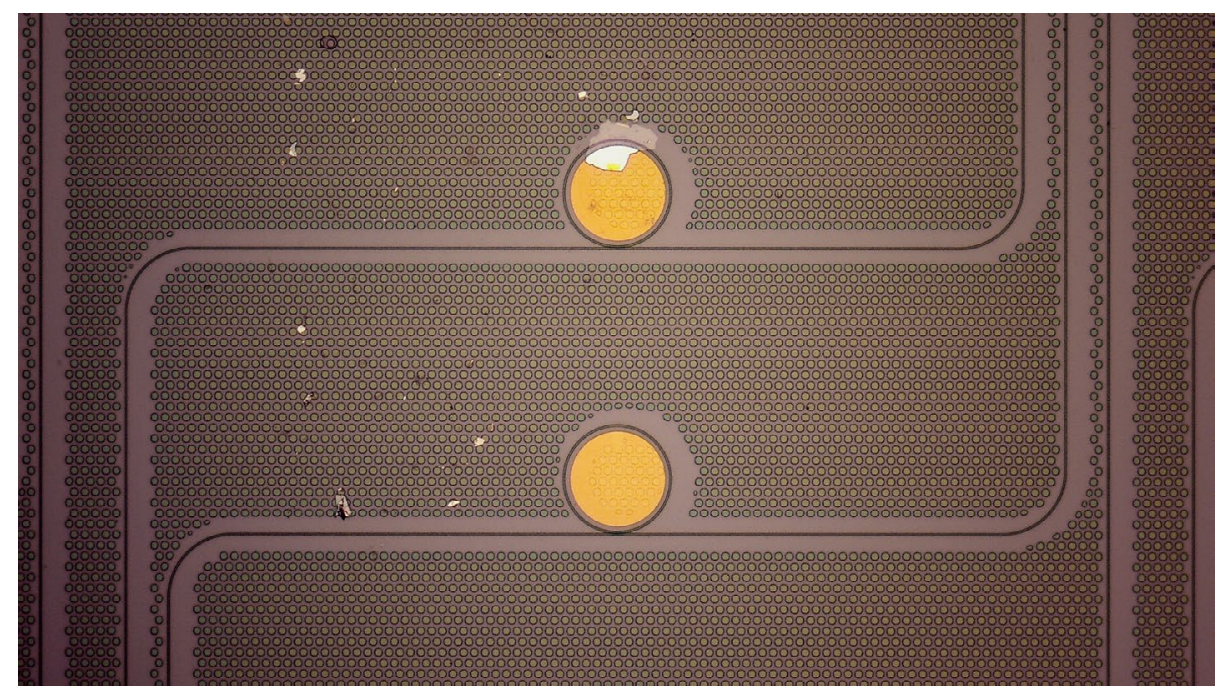

Optical microscopy image of two silicon nitride microring resonators, with the top resonator incorporating a transparent electrode made from graphene doped with a monolayer of tungsten oxyselenide. Credit: Ipshita Datta, Columbia University.

light ${ }^{2}$. With this approach, the polarity of the molybdenum ditelluride and tungsten diselenide channels can be reconfigured from n-type to $\mathrm{p}$-type using laser light of different frequencies. The behaviour is the result of particular light-lattice interactions (such as the formation of self-interstitial defects or the incorporation of substitutional oxygen into vacancies) that occur at different photon energies. The researchers - who are based at the Institute for Basic Science in Korea, Pohang University of Science and Technology, and Seoul National University - used the approach to create a CMOS device that could be reconfigured from an inverter to a switch.

These advances in two-dimensional doping continue in this issue of Nature Electronics, where James Hone, James Teherani and colleagues report that graphene can be doped using a monolayer of tungsten oxyselenide. The monolayer is created by oxidizing a layer of tungsten diselenide, and when the graphene is in direct contact with the tungsten oxyselenide it exhibits a mobility of $2,000 \mathrm{~cm}^{2} \mathrm{~V}^{-1} \mathrm{~s}^{-1}$. By inserting tungsten diselenide layers between the tungsten oxyselenide and the graphene, the carrier density can be tuned and the mobility increased, leading to a mobility of around $24,000 \mathrm{~cm}^{2} \mathrm{~V}^{-1} \mathrm{~s}^{-1}$ with four interlayers. The researchers - who are based at Columbia University, Sungkyunkwan University, the City University of New York, and the National Institute for Materials Science in Tsukuba - also show that the doped graphene is highly transparent in the infrared and illustrate its potential by using it as a transparent conductor in a silicon nitride photonic waveguide and ring resonator.

These examples highlight the range of alternative doping methods that are now available for two-dimensional materials and van der Waals heterostructures, techniques that are helping to create an ever expanding range of sophisticated devices and simple circuits. But in the continuing development of two-dimensional electronics, what demands increasing attention is the fabrication of integrated circuits. This is a topic we will return to next month. Stay tuned.

Published online: 22 October 2021 https://doi.org/10.1038/s41928-021-00668-9

\footnotetext{
References

1. Lee, D. et al. Nat. Electron. 4, 664-670 (2021).

2. Seo, S.-Y. et al. Nat. Electron. 4, 38-44 (2021).
} 